Chirurg 2010 $81: 5-6$

DOI 10.1007/s00104-009-1821-x

Online publiziert: 11. Dezember 2009

c) Springer-Verlag 2009

\author{
H. Bauer \\ Deutsche Gesellschaft für Chirurgie, Berlin
}

\section{Chirurgische Weiterbildung aus Sicht der Deutschen Gesellschaft für Chirurgie}

\author{
Wir wissen, was zu tun ist. \\ Wir müssen tun, was wir wissen
}

Mangel und Krise waren die Schlagworte, die zum Jahreswechsel die politische und gesellschaftliche Diskussion, aber auch die Einschätzung der Zukunft des ärztlichen Nachwuchses und hier insbesondere in der Chirurgie geprägt haben. Vertraut man der Einsicht Max Frischs, dass die Krise an sich ein produktiver Zustand sei und man ihr nur den Beigeschmack der Katastrophe nehmen müsse, sollten vor allem die Chancen gesehen werden, die sich bei wachsendem Handlungsdruck für neue Impulse zu Veränderung ergeben. Bezogen auf die Sicherung von qualifiziertem Nachwuchs in der Chirurgie bedeutet dies neben Veränderungen der allgemeinen Rahmenbedingungen für die ärztliche Arbeit vor allem, die Attraktivität der Weiterbildung zu verbessern.

\section{Defizite zwingen zum Handeln}

In zahlreichen Diskussionen und Publikationen werden derzeit Defizite in der chirurgischen Weiterbildung beklagt. Sie gelten als eine der Hauptursachen dafür, dass sich immer weniger Studienabgänger für das Fach Chirurgie entscheiden, wobei neben einer Untergewichtung chirurgischer Lehrinhalte während des Studiums vor allem Negativerfahrungen im chirurgischen Tertial des Praktischen Jahres eine Rolle spielen. Die erlebte Alltagsrealität chirurgischer Assistentinnen und Assistenten während ihrer klinischen Weiterbildung wird, wie Befragungen zei- gen, im Vergleich zu anderen Fächern offensichtlich als besonders abschreckend und demotivierend empfunden.

\section{Strukturelle und inhaltliche Änderungen sind für eine qualifizierte Weiterbildung notwendig}

Es wäre falsch, dies alles nur den erwähnten Rahmenbedingungen zuzuschreiben, mit denen sich die chirurgiespezifischen Defizite nicht erklären lassen. Unser Problem liegt zum Teil darin, dass wir, sicher unterschiedlich in den einzelnen Facharztqualifikationen innerhalb des Gebiets Chirurgie, keine am Versorgungsbedarf orientierte, Zukunftsperspektiven eröffnende und realistisch umsetzbare Weiterbildungsordnung haben. Deren Anpassung mit notwendigen strukturellen und inhaltlichen Änderungen vor allem in den Facharztsäulen Allgemeinchirurgie, Viszeralchirurgie und Orthopädie/Unfallchirurgie wird gemeinsam von allen unter dem Dach der Deutschen Gesellschaft für Chirurgie (DGCH) vereinten Fachgesellschaften von den für die Umsetzung verantwortlichen Ärztekammern mit Nachdruck gefordert.

Realistische Vorgaben der Weiterbildungsordnung und ihrer Richtlinien sind aber nur die eine Seite der Medaille. Viele Umfragen unter den Weiterzubildenden bestätigen, dass Defizite vor allem in der Planbarkeit, der strukturellen Gliederung, der Qualität der Vermittlung der Inhalte und der Transparenz der Weiterbildung bestehen. Vor diesem Hintergrund ist es allerdings enttäuschend, dass sich, aus welchen Gründen auch immer, nur ein Drittel aller Assistentinnen und Assistenten an der in diesem Jahr erstmals gestarteten bundesweiten Evaluation der Weiterbildung beteiligt haben. Tatsache ist, dass ein erheblicher Teil der Unsicherheit und Unzufriedenheit gerade in unserem Fach auf einer mangelnden Lehrkultur beruht. Die Vermittlung qualifizierter Weiterbildung muss einen höheren Stellenwert bei den dafür verantwortlichen Weiterbildern bekommen. In der Chirurgie fühlen wir uns im Vergleich $\mathrm{zu}$ anderen Fächern dadurch privilegiert, dass wir operieren, also dass wir durch aktives Eingreifen in die Köperintegrität unserer Patienten den Heilerfolg erzielen. Deshalb sind für unser Fach auch spezifische Lehr- und Lernformen und besondere didaktische Methoden zur Vermittlung von manuellen Fertigkeiten von prioritärem Interesse.

\section{Moderne chirurgische Weiterbildung in einem dynamischen Umfeld}

Es ist das Verdienst der Herausgeber und der Autoren, moderne Methoden der chirurgischen Weiterbildung und neue Angebotskonzepte in den Fokus dieser Ausgabe zu stellen. Weiterbildungspro- 
gramme einer leistungsstarken und dadurch auch attraktiven Chirurgie haben nicht nur Veränderungen ganz allgemein in der Medizin und in der chirurgischen Technik zu berücksichtigen. Sie müssen auch einem Wandel in den Arbeitsbedingungen und in den Bedürfnissen der Weiterzubildenden gerecht werden.

Unter diesem Aspekt sind auch die fünf Beiträge zum Leitthema moderner Weiterbildung in der Chirurgie zu sehen. Für die elektronische OP-Lehre, verbunden mit den interaktiven Möglichkeiten des Weblogs (C. Pape-Köhler et al.) gilt, dass bei Evaluierung der derzeitigen Angebote der wissenschaftliche und inhaltliche Gehalt sowie die angestrebte Interaktivität noch deutlichen Verbesserungsbedarf zeigen. Chirurgisches Training am Simulator/Virtual Reality (K. Maschuw et al.) hat sich bei Verbesserung der Systeme und Programme zu einem Übungsmodell mit signifikanten Lerneffekten bei messbarer Steigerung und reproduzierbarer Überprüfbarkeit der manuellen Fertigkeiten entwickelt. Die damit ermöglichte Verlagerung der Lernkurve ist auch das Ziel der seit Jahren fest etablierten „Davos-Kurse“ (H.-D. Saeger et al.), deren bald schon 3 Jahrzehnte dauernde Erfolgsgeschichte auf einem hohen Standardisierungsgrad der Lehrmethode und einer kontinuierlichen Anpassung des Kursangebotes beruht. C. Krones et al. stellen mit dem Aachener Modell ein umfassendes und damit äußerst ambitioniertes Konzept vor, das mit seinen Kursen und Kurrikula durchgängig von der studentischen Lehre und Ausbildung bis zu einer praxisorientierten Weiterbildung neue Wege geht. Der damit verbundene enorme Einsatz macht einmal mehr deutlich, dass neben hoher Motivation aller Beteiligten eine ausreichende Finanzierung der Weiterbildung unabdingbar ist (W. Schröder und K. Welcker). Hier praktikable und auch transparente Lösungen zu finden, bleibt eine der großen Herausforderungen.

\section{Gute Weiterbildung braucht Ressourcen}

Eine gute Weiterbildung ist wesentlicher „Treiber“ einer Arbeitsplatzzufriedenheit im Krankenhaus. Die Anforderungen insgesamt und der Zwang zu Veränderungen haben zugenommen. Ein bereits manifester und künftig sich verstärkender Nachwuchsmangel wurde zum ausschlaggebenden Motor für Veränderungen. Investitionen in die Mitarbeiter lohnen sich, ein Credo, das vor allem private Konzerne auf dem Weiterbildungssektor konsequent verfolgen. Werbung der Kliniken mit attraktiven Weiterbildungsangeboten und Nachwuchskampagnen schon bei Studierenden werden aber nur dann Erfolg haben, wenn Anspruch und Realität nicht weiter auseinanderklaffen. Vom Bundesverband der Medizinstudierenden wird zwar ein merkliches Bemühen konstatiert, den Studierenden etwas zu bieten, von einem echten Bieterwettstreit in Fragen der Aus- und Weiterbildungsqualität könne aber noch lange keine Rede sein.

Sicher ist: Gute Weiterbildung braucht Zeit. Sie braucht Zeit für Zuwendung, Anleitung und Supervision. Sie braucht Zeit für Kommunikation und interaktiven Wissens- und Erfahrungsaustausch. Sie braucht Zeit, um unter Anleitung zu lernen und, was in besonderer Weise natürlich für die Chirurgie gilt, das Handwerk zu üben. Moderne Weiterbildungskonzepte und auch die Nutzung neuer Trainingsmodelle benötigen deshalb neben einem nicht unbeträchtlichen Investitionsaufwand Zeit und damit eine Ressource, die bei den heutigen immens verdichteten Arbeitsabläufen und knapp kalkulierten Personalständen kaum ausreichend zur Verfügung steht. Eine inadäquate Vergütung des Mehraufwands für Weiterbildung ist und bleibt damit ein virulentes Problem. Wertschöpfung darf nicht nur ein heute dominierender Begriff für möglichst rationell erbrachte Krankenversorgung sein. Weiterbildung eines qualifizierten und motivierten Nachwuchses ist eine Wertschöpfung, die jeglicher Anstrengung wert sein sollte.

\section{Fazit}

Es bedarf zahlreicher neuer Impulse im Rahmen einer modernen chirurgischen Weiterbildung. Die Neustrukturierung der Weiterbildungsordnung, wie sie derzeit von der DGCH mit ihren Fachgesellschaften gefordert wird, ist nur ein Teil davon. Es gilt, unter Nutzung innovativer Konzepte neue Wege zu gehen, wie sie in den Beiträgen dieses Heftes dargestellt werden. Gute Weiterbildung muss vor allem einen viel höheren Stellenwert im Selbstverständnis der verantwortlichen Chirurgen erhalten. Mit Larmoyanz und ständigem Klagen über die Rahmenbedingungen werden wir den Nachwuchs nur noch weiter abschrecken. Es gibt vieles, was wir, wie auch hier gezeigt, in die eigenen Hände nehmen können. Damit wissen wir, was zu tun ist. Wir müssen aber auch tun, was wir wissen.

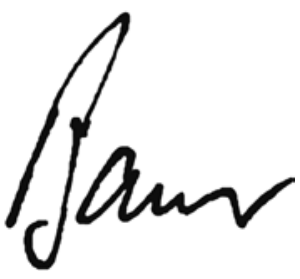

Prof. Dr. H. Bauer

\section{Korrespondenzadresse}

Prof. Dr. H. Bauer

Deutsche Gesellschaft für Chirurgie, Luisenstraße 58/59, 10117 Berlin

H.Bauer@dgch.de 